\title{
Interkulturelle Annäherung im Zeichen der Exogamie
}

\author{
Kommunikationsstrategien im König Rother
}

\section{Amelie Bendheim}

\begin{abstract}
Concerned about the royal succession, the powerful King Rother follows the advice of his nobles and sends a group of envoys to the foreign country of King Constantine to seek the hand of his beautiful daughter. This article aims to discuss the concept of >dangerous courtship as an intercultural contact scheme. It describes the relation between divergent communication practices and different forms of sovereignty. As a Middle High German text which does not address interculturality using the usual paradigms of demarcation (e.g. the interreligious dichotomy of Christians vs Heathens), König Rother reveals a broad spectrum of intercultural questions not yet discussed in the context of medieval narration.
\end{abstract}

Title: Intercultural Approximation and Exogamy - Communication Strategies in König Rother

Keywords: medieval culture; interculturality; courtship; exogamie; communication

Der Titel des folgenden Beitrags mag zunächst widersinnig anmuten, ist ihm doch das Anliegen zu entnehmen, den König Rother für die interkulturelle Mediävistik fruchtbar zu machen: Gerade den König Rother, mag sich manch einer denken, wo sich doch auf den ersten Blick stilistisch wie poetisch bessere, schönere und noch dazu >interkulturellere< Texte zur Behandlung des Themas finden ließen. Schließlich kann die anonyme, aus dem 12. Jh. stammende Verserzählung' zumindest insofern nicht als Glanzstück mittelalterlichen Erzählens gelten, als sie konventionelle rohe Schemata und Motive (wie das der gefährlichen Brautwerbung) verarbeitet, die, zwar mit zeitgenössischen Bezügen gefüllt, ${ }^{2}$ in eine stilistisch >hölzerne< Erzählform eingepasst werden. Der Handlungsverlauf wird dem Rezipienten in sprunghaft herausgelösten Einzelbildern - ähnlich einer Guckkastentechnik - präsentiert und unterscheidet sich damit deutlich

1 Über die genaue Datierung des Rother herrscht in der Forschung Uneinigkeit: Die traditionelle Datierung auf die Jahre 1150 bis 1160 (so Szklenar 1986: 89) wird von Bumke (vgl. 1979: 92/335f.) als zu früh angesehen, der von einer Entstehung zwischen 1152 und 1180 ausgeht.

2 | Szklenar betont, dass Themen verarbeitet wurden, "die ein adeliges Publikum im 12. Jhd. nicht nur als Elemente serzählterı, sondern eigener Wirklichkeit interessieren konnten" (Szklenar 1986: 91). 
von den Organisationsformen großepischer Werke, wie sie in der Zeit hochhöfischen Erzählens das poetologische Schaffen von Dichtern wie Wolfram von Eschenbach oder Gottfried von Straßburg charakterisieren. ${ }^{3}$

Und auch unter dem Aspekt der Interkulturalität standen bis dato eher andere mittelhochdeutsche Texte - insbesondere jene der Chanson-de-Geste-Tradition - im Fokus wissenschaftlicher Betrachtung, Texte, die kulturelle Gegensätze und Spannungen offener, nämlich zumeist im deutlich hervortretenden Religionskonflikt (Christen versus Heiden) behandeln bzw. in der Überwindung solcher Konflikte durch Toleranz ein >differenzierteres Heidenbild< vermitteln. ${ }^{4}$

Die Auswahl des Brautwerbungsepos König Rother (KR) als interkultureller Analysegegenstand ist daher auch eine Entscheidung gegen eine solch starke Zentrierung auf Erzählstoffe der Chanson de Geste und den Aspekt religiöser Differenz sowie für eine stärkere Offenlegung des breiten Spektrums interkultureller Themen im Rahmen des mittelalterlichen Erzählens. ${ }^{5}$ Der Beitrag richtet den Fokus dabei auf drei Leitfragen, die den Aufsatz in der folgenden Reihung strukturell gliedern: Erstens, wie gelingt es, über das Brautwerbungsschema kulturell verschieden kodierte Erzählwelten zu öffnen, und wie konstituieren sich diese Welten im KR? Zweitens, wie wird der Kontakt zwischen diesen Welten narrativ gestaltet, und zu welchem Ende wird er erzählt? - Hier interessiert besonders die Frage: Wie wird mit der >unterlegenen Kultur < umgegangen? Drittens und abschließend: Was bedeutet es für die KR-Rezeption, ihn als >interkulturelles Zeugnis< zu lesen, d.h., welches Textverständnis wird erworben, wenn wir uns damit befassen, wie er mit Interkulturalität umgeht?

\section{ERzÄhlWelten IM KöNIG RotheR}

Das Erzählschema der gefährlichen Brautwerbung (vgl. hierzu v.a. SchmidCadalbert 1985: 79-100) schreibt vor, dass der Held in die Fremde ziehen muss, um dort eine Braut für sich zu gewinnen, dass er bei diesem Versuch eine Ge-

3 | Sprachlich-stilistisch zeichnet sich der Rother durch eine große Füllungsfreiheit und starken Assonanzgebrauch aus (vgl. ebd.: 90), es dominiert die einfache Darstellungsform der Addition, einer szenisch-ausschnitthaften Betrachtung. Fromm spricht diesbezüglich von "szenische[r] Kleinräumigkeit" (1976: 373) und verweist darauf, dass diese ein Merkmal frühmittelhochdeutscher Bibelepik und der Chanson de Geste darstelle.

4 | Etwa in Wolframs von Eschenbach Werken Willehalm und Parzival; vgl. hierzu beispielhaft einige der zur Thematik entstandenen Beiträge, die den Aspekt der religiösen Toleranz besonders fokussieren: Jackson 1969; Ebenbauer 1984; Samples 2001; Haupt 2009; Schotte 2009.

5 | Auch im Rother liegt zwar ein Religionskonflikt vor, der in der Behauptung des Christentums gegenüber dem Islam (verkörpert von der Figur des Heiden Ymelot) kämpferisch ausgetragen wird. Allerdings wird er auf einen Nebenschauplatz verlagert und bildet nicht das Zentrum der interkulturellen Spannungen zwischen Rother und Konstantin. 
genfigur (meist den Vater der Braut) besiegt, mit der Braut zurückkehrt und die Herrschaft sichert. Auch im KR wird diese, dem mittelalterlichen Erzählen vertraute Strukturschablone angelegt: Exogamieregel und Raumstruktur führen dabei zur Annäherung zweier unterschiedlicher (Be-)Reiche, dem westlichen Herrschaftsbezirk König Rothers (in Bari, am adriatischen Meer) und dem östlichen Kaiser Konstantins (in Konstantinopel, Griechenland). ${ }^{6}$

Um das Bezugsverhältnis zwischen diesen nicht nur geographisch, sondern insbesondere auch kulturell (und auf einer anderen Ebene als der des Glaubens, denn beide Herrscher sind Christen) entfernt voneinander situierten Räumen definieren zu können, soll folgend mit einem relativ weiten (semiotisch geprägten) Kulturbegriff gearbeitet werden. Er begründet sich in der älteren Kulturphilosophie, und hier insbesondere durch Ernst Cassirers Hauptwerk Philosophie der symbolischen Formen (vgl. Cassirer 2010), in dem Kultur als ein Konstruktionsprozess begriffen wird, der bestimmte Zeichen- und Bedeutungssysteme hervorbringt.7 Kultur, menschliche Kulturleistungen oder Kulturbereiche, offenbart sich als Menge von symbolischen Formen (der Form des Denkens, des Mythos, der Religion, der Wissenschaft), die ein Netzwerk von Zeichenhandlungen bilden. Sie erzeugen, so auch die kulturwissenschaftliche Forschung nach Clifford Geertz, ein »selbstgesponnene[s] Bedeutungsgewebe« (1987: 9). ${ }^{8}$ Pragmatisch ausgelegt (und für den hier behandelten konkreten $>K R$-Fall<) bedeutet dies, dass im Spiegel der Interkulturalität insbesondere das Kontakt- und Konfliktpotenzial unterschiedlicher Herrschaftsformen aufgedeckt und damit die >politische Kultur<als spezifische Ausprägung eines kulturellen Bedeutungsgewebes behandelt werden soll. Dabei, so die Ausgangsthese, offenbart sich Kultur im $K R$ wesentlich über je spezifisch geprägte Sprechweisen sowie in divergierenden Kommunikationsstrategien, mit denen sich Herrschaft artikuliert und Rechtsprechung vollzieht.

6 | Beide Ortsangaben nehmen einen prominenten Platz ein: Bi deme westeren mere (KR 1), "An der Küste des Adriatischen Meeres", als Eröffnung der Erzählung, und auch die Lokalisierung des Reiches Konstantins geht noch der Erwähnung der Königstochter voraus (ich weiz [...] oster over se / einis riken kuninges tôchter vil her, KR 64-66, "ich weiß im Osten jenseits des Meeres von der vornehmen Tochter eines mächtigen Königs").

7 | Vgl. dazu Ort 2003, hier bes. Pkt. 3 (Kulturtheorie als Zeichentheorie) sowie in der Einführung von Bauer 2005, hier bes. Kap. 2.3 (Kultur als Netzwerk von Zeichenhandlungen).

8 | Geertz folgert weiter, dass die Untersuchung von Kultur daher "keine experimentelle Wissenschaft [ist], die nach Gesetzen sucht, sondern eine interpretierende, die nach Bedeutungen sucht“ (Geertz 1987: 9). Vgl. dazu auch Ernst Cassirer, Versuch über den Menschen (1990: 49-51): "Der Mensch entkommt dieser seiner Erfindung nicht. Er lebt nicht mehr in einem bloß physikalischen, sondern in einem symbolischen Universum. Sprache, Mythos, Kunst und Religion sind Bestandteile dieses Universums [...]. Der Begriff der Vernunft ist höchst ungeeignet, die Formen der Kultur in ihrer Fülle und Mannigfaltigkeit zu erfassen. Alle diese Formen sind symbolische Formen". 
Um diese am Text zu illustrieren, soll zunächst erneut auf den erzählerischen Anfang Bezug genommen werden: Schemakonform setzt der KR mit einer Beratungsszene am Hof ein, die bereits die Grundstrukturen der Kommunikation des Königs offenlegt: Gegenstand der Diskussion ist die anvisierte Brautwerbung, die dem Herrscher eine geeignete Dame zuführen und damit eine gesicherte Thronfolge begründen soll (vgl. KR 29-32). Rother bekennt diesbezüglich und vorab zwar, im eigenen Land schon selbst >Ausschau gehalten $<$ und auch bereits einige weibliche Bekanntschaften gemacht, aber keine geeignete Brautanwärterin gefunden zu haben, die mir so wol gevalle / daz ir sie lobit alle (KR 43f.). 9 Es folgt der Bericht des Grafen Lupold, der von einer Dame in der Ferne kündet, die luchtit uz deme gedigene / so daz gesterne tovt vun deme himele (KR 71f.), ${ }^{10}$ und die Rother fortan zu gewinnen erstrebt. Auch der anschließende Rat des Markgrafen Hermann, wer als Bote die Reise für den Herrscher antreten könnte (vgl. KR 85f.), wird von Rother berücksichtigt und trägt dazu bei, die Brautwahl deutlich als konsensualen Ratsbeschluss auszuweisen. ${ }^{11}$

Es ist dies das erste, bei weitem aber nicht das einzige Mal, dass Rother sich der Entscheidung seines Rates fügen wird: ${ }^{12}$ Zwölf Rother-Beratungsszenen (mit einem Umfang von insgesamt ca. 500 Versen) durchziehen in kontinuierlicher Folge das Epos, das in der annähernd vollständigen Handschrift $\mathrm{H}^{13}{ }_{5181}$ Verse umfasst, und nehmen damit den beachtlichen Anteil von rund zehn Prozent des gesamten Textvolumens ein. Mehrfache Repetition der Beratungsszenen also zum einen, die zum anderen auch durch ihre formale, nämlich szenische Präsentation mittels wörtlicher Figurenrede hervorgehobenen Status erlangen. ${ }^{14}$ Sie erzeugen beim Publikum eine unmittelbare Nähe zum erzählten Geschehen (vgl. Martínez/Scheffel 2012: 49) und rücken das Kommunikationsverhalten des Herrschers mit distanzlosem Nachdruck in den Fokus. ${ }^{15}$

9 | "Die meinen Bedingungen so entspräche, daß (auch) ihr alle mit inr einverstanden wäret".

$10 \mid$ "[A]us dem Gefolge hervor[leuchtet], wie es der Morgenstern vom Himmel herab tut".

11 | Die Wortwahl Brunners, der inhaltlich resümiert, "die Wahl fällt auf die Tochter des Königs [...] Konstantin“ (Brunner 2017: 138; Hervorh. A.B.), scheint diesbezüglich nicht ganz glücklich, insofern sie dem Prozess eine Unvermitteltheit unterstellt, die inm gerade nicht zukommt.

12 | Auch Meves verweist darauf, dass Rother nicht an die Vorschläge des Rates gebunden ist, sich als Herrscher aber gerade dadurch hervortut, dass er seine Machtposition nicht ausnutzt, um eigenmächtig zu agieren (vgl. Meves 1976: 29).

13 | Zur näheren Information über den Bestand der Handschriften vgl. Szklenar 1985: Sp. 82.

14 | Die szenische Erzählform setzt die Präsenz des Erzählers auf null und erzeugt durch die Verkürzung der Distanz zum Erzählten unmittelbare Nähe zum Geschehen und größtmögliche rmimetische Illusion` (vgl. Martínez/Scheffel 2012: 49).

15 | Näher einzugehen wäre diesbezüglich auch auf die sprachliche (insbesondere morpho-semantische und pragmatische) Gestaltung der Szenen, der sich eine ex- 
Im Rother'schen Reich bildet der königliche Rat ${ }^{16}$ ein Herschaftsorgan, das zur Entscheidungsfindung beiträgt, indem divergierende Meinungen und Argumente (vor dem Hintergrund gesellschaftlicher, politischer wie auch persönlicher Interessen) ausgetauscht werden: Da horde man manigin vromen man / vromicliche rede han, / da mide sie ir herren / hulfen grozer erin (KR 552-55) ${ }_{17}^{17}$ heißt es so oder in ähnlicher Formulierung im resümierenden Erzählerkommentar derartiger Redeszenen. Besonders deutlich tritt die Motivation Rothers, eine konsensuale Lösung zu erzielen, nach der gescheiterten ersten Brautfahrt nach Konstantinopel hervor, die die Gefangennahme und Einkerkerung der Rother'schen Gefolgsleute zur Folge hatte. So ergreift zunächst Berchter, der erste Berater Rothers, das Wort und rät unmittelbar zum Gegenangriff, indem er einen gewaltsamen Auszug mit großem Heer fordert, schließlich hat er bereits den Verlust einiger Söhne durch Kriegszüge zu beklagen ${ }^{18}$ und fürchtet nun auch um das Leben seiner sieben anderen Kinder, die als Boten zur Brautwerbung aufgebrochen waren. Rother aber belässt es nicht bei dieser einen (vorläufigen) und persönlich motivierten Meinung, sondern bezieht alle am Hof befindlichen Ratgeber in die Entscheidungsfindung mit ein:

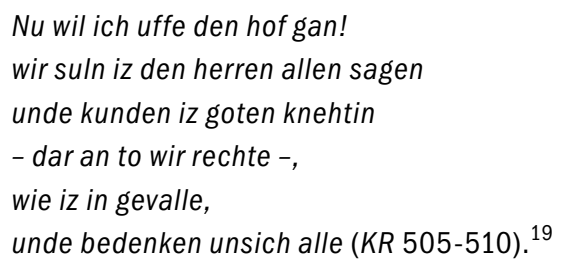

plizite Verwendung von ratsı-Vokabular nachweisen lässt (vgl. beispielhaft: sinen rat nam (KR 459), du salt mir ratin (KR 462), daz sal nu min rat sin (KR 493), an den rat gan (KR 549), si ir herren / hulfen (grozer erin) (KR 555), si reiten (iren herren) (KR 558), nicht bezzeris ratis vinden (KR 605), gerne volgan (KR 611), volge dem rade $\min (K R$ 5156), volge mir (KR 5168; alle Hervorh. A.B.). Auch durch die spezifische Verwendung imperativer Verbformen (du salt, KR 4616, hier sal, KR 4632) in diesen Abschnitten artikulieren sich direktive Sprechakte mit dem Zweck der zukünftigen Handlungsverpflichtung.

16 | Dieser Rat setzt sich aus den namentlich genannten Figuren Berchter (dem engsten Berater) und Lupold (dem treuen Gefolgsmann und Verwandten Rothers) sowie der im Plural erfassten klassischen adeligen Gefolgschaft (thuren volcdegene, KR 57, "die vortrefflichen, im ganzen Volk berühmten Helden", sine man, KR 2665, "seine Mannen ", herrin allin sammt, $K R$ 3339, "die versammelten Edlen", manich got knecht, $K R$ 3351, "viele vortreffliche Gefolgsleute", vrunt inde man, KR 3666, „Freunde und Gefolgsleute") zusammen.

17 | "Da hörte man viele vortreffliche Leute Nützliches vorbringen, womit sie ihrem Herrscher zu einer ehrenvollen Lösung des Problems verhalfen."

18 | [M] ich ruwent sere mine kint, KR 497, "der Verlust meiner Kinder schmerzt mich sehr".

19 | "Jetzt aber will ich noch zur Hofversammlung gehen! Wir müssen es (auch) allen Herren (des Rates) vortragen und den tapferen Rittern bekanntmachen - damit han- 
Dass es sich hier um eine institutionalisierte Form strukturierter Kommunikation handelt, macht Rother deutlich, wenn er mit dem Mittel der Parenthese, d.h. syntaktisch von der Versumgebung losgelöst und dadurch zusätzlich gewichtet, auf die Rechtmäßigkeit des Vorgehens verweist (vgl. KR 508). Das Kommunikationsorgan des Rates erscheint als sinnstiftende Institution sowohl gegenüber einem unsicheren Schicksal (vgl. $K R$ 518f. ${ }^{20}$ ) wie auch gegenüber der alleinigen Entscheidungsgewalt eines einzelnen Herrschers, die Rother selbst in Zweifel zieht: »Was wäre, wenn es da [im Rat; A.B.] jemanden gibt, der einen besseren Plan hat als den, den wir uns zurechtgelegt haben? ${ }^{21}$

Das Kommunikationsverhalten von Rothers Konkurrent Konstantin unterscheidet sich davon fundamental: Dem polyperspektivischen Einbezug von Argumenten in die Entscheidungsfindung Rothers treten Konstantins Reden »in monologischer Verkapselung « (Fromm 1976: 375) gegenüber: Im Kontext bevorstehenden Unheils begegnet er der Warnung seiner Boten vor dem Angriff des heidnischen Heeres unter Ymelot zwar ebenfalls mit einer Frage, die hier als rhetorisches Redemittel jedoch lediglich selbstreferentielle Überlegenheitsdemonstration behauptet: Wer mochte so riche sin, / der mich torste bestan? (KR 2588f.). ${ }^{22}$ Der östliche Herrscher sieht sich im Alleinbesitz der Wahrheit, als (maßlos) über das Wort gebietend und mit diesem unmittelbar handelnd: So ordnet er unter Androhung des Galgens an, dass sich ein jeder zum Hoffest für die Tochter einzufinden habe:

her hiez sie sichriliche varen

do mostin si alle dare.

swer sich ieht sazte dar widir,

deme gebot man iz bi der widen,

daz her gerner dar gienge,

dan man in hienge.

do ne torstiz nieman irlan. (KR 1571-1576) $)^{23}$

Recht auf Widerspruch wird auch der eigenen Tochter nicht zugestanden, deren Gefühlsregungen, sie qualite ir lib (KR 3849), ${ }^{24}$ nach der Übergabe an den heid-

deln wir dem Recht entsprechend (um zu sehen,) wie es innen gefällt, und uns gemeinsam beraten."

20 | [W]az, ob sie [referiert auf die Gefährten; A.B.] der grimige tot / noch hat neiht bevangin?, "Was ist, wenn sie der schreckliche Tod noch nicht in seiner Gewalt hat".

21 | [W] at ob itthelicher ist, / der hat bezzere list / dan wir uns haben genumen, KR 511-513.

22 | "Wer könnte so mächtig sein, daß er es wagte mit mir den Kampf aufzunehmen?"

23 | "Er verpflichtete sie streng zum Kommen, da mußten sie alle dorthin [...]. Jedem, der auch nur das geringste Widerstreben zeigte, befahl man es unter Androhung des Galgens, damit er es eher vorziehe hinzukommen, als gehängt zu werden. Da wagte es niemand zu unterlassen."

24 Sie "marterte sich (mit inrem Schluchzen)". 
nischen Herrscher Ymelot nicht zur Kenntnis genommen: $n u$ swic, tochter min (KR 3851). ${ }^{25}$ Die bekundete Alleinherrschaft über das Wort zwängt Konstantin in festgefahrene Handlungsmuster, die auch im wiederholt verwendeten Verb stan/bestan plastisch hervortreten: stan muss Konstantin lange trorich $(K R 327)^{26}$ und bestan (KR 1814) $)^{27}$ muss wiederholt auch sein zorn (ebd.). ${ }^{28}$ Dass körperliche Regungslosigkeit ${ }^{29}$ mit geistiger Unbeweglichkeit einhergeht, zeigt sich auch bei der Eskalation im Festsaal, wenn der Riese Asprian (ein Gefolgsmann Rothers) den >Hauslöwen < Konstantins an die Wand schmettert, ${ }^{30}$ worüber der Herrscher sich zwar betrübt zeigt, jedoch - handlungsunfähig - noch nicht einmal in der Lage ist, seine Füße zu bewegen: we leide eme der kuninc do saz, / her ne geregite doch nie de vote (KR 1153f.). ${ }^{31}$ Die Atmosphäre dieses negativen Stillstands bekommen auch die Gefolgsleute Konstantins zu spüren, deren Bitte, die Gefangenen aus dem Kerker zu entlassen, nicht erhört wird (ir bete were al verloren, $K R$ 1201). Sie müssen den herrscherlichen Unmut schlicht erdulden (se mostin dolen sinen zorn, KR 1201f.), ${ }^{32}$ was zu Kritik führt, die sich in ungeschönter Direktheit kundtut: Du hast den valant getan! (KR 890), ${ }^{33}$ schelten sie Konstantin. ${ }^{34}$

Auch Rother wird als Herrscher in statischer Position, auf einem Stein sitzend (vgl. KR 448), abgebildet, in der er sich selbst aus Trauer das Recht auf Rede untersagt (dre tage unde drie nacht, / daz er zo niemanne nicht ne sprach,

25 | "Schweig jetzt, meine Tochter!", s. auch nach der Rückentführung der Tochter aus Rothers Land: her inhatte uf ire sprechin nit. / he liez si swigin unde dagin, KR 3266f., "er wartete nicht darauf, daß seine Tochter redete, er ließ sie in inrem beharrlichen Schweigen gewähren ". Zur Form der hier praktizierten Eheschließung, die dem dynastischen Prinzip der Munt-Ehe folgt, bei der der Ehevertrag zwischen dem Bräutigam und dem Gewalthaber geschlossen wird vgl. Schott 1986.

26 | "[L]ange mit Kummer".

27 | "[B]estehen bleiben".

$28 \mid$ "Unmut".

29 | Sie offenbart sich auch in der Reaktion auf die Entführung der Tochter, die inn vor Leid in Ohnmacht fallen lässt (vgl. KR 3015). Konstantins Regungslosigkeit wird hier durch die vorangestellte Konjunktion unmittelbar mit dem aktiven Handeln Ymelots kontrastiert, der die Stadt verlässt (do Constantin darnider lach / Imelot huf sich uze der stat, KR 3034f., "Während Konstantin ohnmächtig dalag, machte sich Ymelot aus der Stadt davon").

30 | Asprian begreif ene [den Löwen; A.B.] mit der hant / unde warf ene an des sales want, / daz her al zebrach, KR 1150-1152, "Asprian packte inn mit der Hand / und schleuderte inn an die Wand des Saales, / daß er daran gänzlich zerschellte".

31 | "Sosehr betrübt darüber der König auch dasaß, / er bewegte dennoch nicht einmal seine Füße." Erst im Nachhinein bekennt er - wenig zielführend -, man hätte direkt mit Gewalt antworten und den Riesen erschießen sollen.

32 | "hre Bitte sei völlig sinnlos, / sie müßten seinen Grimm erdulden".

33 | "Du hast den Teufel getan!"

34 | Die Anklage bezieht sich hier auf die Gefangennahme der Boten, die der König einsperren ließ, ohne sich über die Konsequenzen möglicher Racheakte Gedanken zu machen. 
KR 450f.). ${ }^{35}$ Hier allerdings handelt es sich um einen zeitlich klar begrenzten (drei Tage und drei Nächte) und damit als Ausnahmesituation markierten Zustand der Reflexion, der zudem als Form produktiver Passivität zu begreifen ist: Denn der nach innen gerichteten Denkbewegung folgt unmittelbar eine Öffnung nach außen und die Umsetzung in bzw. Forderung nach körperlicher Aktivität: Berchter soll Rother raten we wir kumen ober mer $\left(K R{ }_{4} 6_{3}\right) \cdot{ }^{36}$ Wohlüberlegt und klug (harde wisliche, $K R 3665)^{37}$ kommuniziert Rother den Seinen (vrunt inde man, $K R \quad 3667)^{38}$ seine Pläne (etwa jenen, nun inkognito, in Pilgerverkleidung, in Konstantinopel einziehen zu wollen). Interaktion, das zeigt sich in der Herrschaft Rothers, ist ein Zeichen von Treue und Loyalität. Sie trägt zur Lösungsfindung bei, die auch die präferierte Wahl einer gewaltfreien und damit zivilisierten Form der Problembewältigung dokumentiert. ${ }^{39}$

Rother erscheint als vorbildlicher Herrscher, dessen Macht auf der wechselseitigen Einflussnahme von Herrscher und Gefolge gründet: ir habit vrumicliche getan, / ich wil u gerne volgan! (KR 610), ${ }^{\circ}$ bezeugt Rother und auch das Gefolge präsentiert sich als Einheit, wenn die Boten beim Auslaufen des Schiffes gen Osten ihre geteilte Gesinnung bekunden, indem sie iren rơf [...] do hoben (KR 180), also einen gemeinsamen Erkennungsruf erschallen lassen.

Das Modell >gut beratener König< vermag als erfolgreich hervorzutreten, wobei die Beratung nicht nur durch ihr (bereits angesprochenes) wiederholtes Auftreten im Werk, sondern auch durch teils skurrile (Realisierungs-)Formen zur Leitfigur erhoben wird: Unter dem Tisch im Festsaal Konstantins versteckt, ruft Rother den gesamten Rat zusammen: ${ }^{41}$ rother der riche / beriet sich eremliche (KR 3930f.), ${ }^{42}$ kommentiert der Text die Szene und setzt im (unreinen) Reimpaar riche-ermeliche zwei kontrastierende Begriffe in eine syntaktisch parallele

35 | Rother saß auf einem Stein "drei Tage und drei Nächte so, daß er mit niemandem sprach".

36 | "Auf welche Weise wir übers Meer fahren sollen."

37 | "[W]ohlüberlegt und klug".

38 | "Freunde und Gefolgsleute".

39 | So auch Jolie (vgl. 2005: 185), der betont, die Entscheidungen zwischen zwei Alternativen werden immer wieder zugunsten der gewaltvermeidenden getroffen. Die archaische Gewalt schwelt im Hintergrund, kann aber durch die Wahl zivilisierter Formen überwunden werden (vgl. ähnlich auch Kiening [1998: 233]: „der Text [wird] gerade vor dem Hintergrund unrealisierter Alternativen profiliert").

40 | "Ihr habt wie gute Ratgeber gehandelt, ich will euch gerne folgen." Vgl. ähnlich auch die folgende Sentenz: ia hortich [...] sprechen / so wer were ein got recke, / daz her unrechte tete, / so man ime goten rat gebe, / daz er des nicht ne neme, KR 500504, "Ich hörte sagen, daß der, der ein vortrefflicher Held sein wolle, falsch handele, wenn er einen guten Rat, den man inm gibt, nicht annehme".

41 | Wie man sich das genau vorzustellen hat, bleibt wohl jedem selbst überlassen, der Tisch muss jedenfalls riesig gewesen sein.

42 | "Der mächtige Rother hielt mit beschränktem Aufwand eine Ratsversammlung ab." 
Reimendstellung, mit denen der Dichter markiert, dass jede noch so ärmliche (hier vor allem räumlich beschränkte) Form der Beratung dem König den immateriellen Reichtum, der dieser Beratung erwächst, nicht abzusprechen vermag, sondern ihn vielmehr noch zusätzlich unterstreicht.

\section{Die transkulturelle Braut - Kontaktaufnahme ZWISCHEN ZWEI WELTEN}

Wie nun aber, so ist zu fragen, kommen die beiden einander derart entgegengestellten Erzähl- und Herrschaftswelten (Rother-Welt/Konstantin-Welt) miteinander in Kontakt? Im Rother verläuft diese Verbindung über einen Mittler:43 Die auserwählte Braut und Tochter Konstantins wird (quasi) zur transkulturellen Kontaktfigur. Sie bleibt namenlos und damit auf ihre Funktion reduziert, sowohl die kulturellen Divergenzen durch wiederholte Grenzüberschreitung sichtbar zu machen als auch ein Hin- und Herblenden zwischen den verschiedenen Herrschaftssystemen zu ermöglichen. ${ }^{44}$

Denn die Braut ist Anlass zur ersten Brautwerbungsfahrt in die Fremde und treibt die Boten an, sich auf hoher See in Richtung Konstantinopel, in das fremde Land (vgl. KR 202), aufzumachen: eilf graven ime do sworren, / daz si erme herren umbe die maget voren (KR 144f.). ${ }^{45}$ Der scheiternden ersten Werbungsfahrt, auf der die Boten gefangengenommen werden (vgl. KR 198-202), schließt sich die zweite Ausfahrt an, die die Entführung der Braut durch Rothers Gefolgsleute zur Folge hat:

ir mogit eme (Konstantin) werliche sagin,

sin tochter si mit Rothere

gevaren westene over mere (KR 2914-2916). ${ }^{46}$

Es kommt dann zur Rückentführung der Königstochter durch den Brautvater Konstantin,

43 | Mit Colin, die verschiedene Typen von Mittlerfiguren (ephemere Mittler, moralisch/politisch diskreditierte Mittler, ambivalente Mittler etc.) genauer differenziert, wäre hier von einer 'nicht-intentionalen Mittlerfigur zu sprechen (vgl. Colin 2013, hier bes. 96f.). Auch mit spezifischem Fokus auf das Mittelalter wäre eine genauere Ausdifferenzierung der interkulturellen Mittlerfiguren erstrebenswert.

44 | Kohnen (2014: 255) führt die zunächst ungewöhnlich scheinende Namenlosigkeit der Figur auf die Bedeutsamkeit ihrer historischen Anbindung zurück, die es ermöglicht, "die Figur [...] mit jeder byzantinischen Prinzessin in Beziehung [zu setzen]" und so auf abstrakter Ebene einen Kontextbezug herzustellen.

45 | "Elf Grafen schworen inm da, daß sie für ihn, ihren Herrn, um die junge Dame ausziehen wollten".

46 | "hr könnt inm dann wahrheitsgemäß sagen, seine Tochter sei mit Rother nach Westen über das Meer gezogen “. 
din wif ist wider over mere!

daz havet Constantinis man

mit grozen listen getan (KR 3302-3304) ${ }^{47}$

und schlussendlich zur erneuten Ausfahrt Rothers, ${ }^{48}$ die zum endgültigen Gewinn der Braut führt: die vorde der koninc Rothere / mit sineme wife over mere (KR 4745f.). 49

In der mittelalterlichen Rother-Erzählwelt, die Kiening zu Recht als eine »Welt der Präsenz der Körper « $5^{\circ}$ bezeichnet, wird der abstrakte Vergleich kultureller Muster somit in unvermittelter Form (nämlich durch die körperlich präsente Mittlerfigur) erzählbar gemacht. Die Braut wird zum Symbol des Kommunikationsprozesses zwischen Ost und West, verleiht dem (narrativ entfalteten) Kontakt zwischen den Kulturen unmittelbare Sichtbarkeit.

Die Interaktion über das Wasser lässt zudem das Meer als Medium der Kontaktherstellung hervortreten, als wertfreien Transitraum, der von beiden Parteien gleichermaßen >bespielt< und durchfahren wird, ohne dass in ihm bereits eine Kontaktaufnahme erfolgen würde. Das Meer im KR ist ein » Nicht-Ort< des ruhelosen Stellungswechsels« (Borgolte/Jaspert 2016: 17), ein bewegliches Element an sich und damit auch als Prozess zu begreifen, der die Strukturen eines (interkulturellen) Gesprächs allererst etabliert. »Wie viele andere Grenzregionen trennen Meere nicht nur«, vermerken Borgolte/Jaspert in ähnlicher Weise zur Funktion des Wassers, »sondern sie dienten auch als Räume erhöhter Verflechtung, weil über sie Waren, Menschen, Ideen und anderes mehr zirkulierten« (ebd.: 22). Die Fahrten über die See sind dabei in doppelter Weise als Moment der Ungewissheit konnotiert: Zum einen ist es das Element des Wassers selbst, das als unberechenbare Naturgewalt dem feststehenden Land

47 | "Deine Gattin befindet sich wieder jenseits des Meeres! Das hat ein Gefolgsmann Konstantins nach einem schlau ausgedachten Plan getan".

48 | Do vorte der koninc Rothere / drizic dusint over mere, [...] / die kiele giengen evene / inde quamen in ses wochen / over mere gevlozzen / hin ze Constantinopole, KR 3632-3642, "Da hatte der König Rother für seine Heerfahrt übers Meer dreißigtausend Mann [...] Die Schiffe zogen gleichmäßig dahin und gelangte, nachdem sie sechs Wochen übers Meer gefahren waren, nach Konstantinopel«.

49 | "[D]iese (Krieger) führte der König Rother zusammen mit seiner Frau über das Meer".

50 | Kiening 1998: 222. Darin erfolgt, so Kiening weiter, der Kontakt verbal, gestisch, körperlich, nicht aber in vermittelter Form." Vgl. dazu auch Peter Czerwinski, der die mittelalterliche Welt als "Welt der vorherrschenden Dinglichkeit" beschreibt, in der Reflexion und Abstraktion nur über ein materielles Referenzobjekt in Gang gesetzt werden können. Gegenwärtigkeit war nicht über einen reinen Denkprozess herzustellen, sondern an ein Konkretes gebunden (vgl. dazu genauer Czerwinski 1989: 22). 
(deme stade, $K R$ 181) explizit entgegengestellt wird. ${ }^{51}$ Das bewegte Meer nimmt die Männer auf (die herrren vluzzin in dat mer, KR 184) und sich ihrer sozusagen richtungsweisend an, wenn sich sofort nach Abfahrt »rauschend die Segel blähen « $\left(K R 1_{182}\right)$ und Rother nichts anderes übrigbleibt, als Gott zu bitten, er möge seine Gefolgsleute wieder wohlbehalten zovlande $\left(K R{ }_{18} 9\right)^{52}$ geleiten. Zum anderen verbindet sich im $K R$ mit dem Über-Meer-Fahren auch die Vorstellung, dass jenseits des Meeres das kulturell Andere lokalisiert ist. ${ }^{53}$ Die mehrmalige Überquerung des Meeres verstärkt und verstetigt den dynamischen Prozess der Kontaktherstellung, macht aber auch deutlich, dass sich der Dialog in einem noch undefinierten und damit immer auch angstbesetzten Übergangsstadium befindet.

\title{
ZU WELCHEM ENDE WIRD DER INTERKULTURELLE KONTAKT IM KÖNIG ROTHER ERZÄHLT?
}

Die zum Ende hin erfolgende Arretierung dieser bewegten Erzählführung geschieht zugunsten einer Fokussierung auf den Herrschaftsbereich Rothers; die Kamera hält an, der Zoom richtet sich auf das Rother-Reich, das nun in uneingeschränkter Vorbildlichkeit erstrahlt. Denn mit der letztlich doch erfolgreich realisierten Brautwerbungsfahrt kann die im Anfang problematisierte und im Text singuläre Leerstelle (er ane vrowen was) in der ansonsten bereits als ehrenhaft gepriesenen Herrschaft des Königs beseitigt werden:

\author{
Rovther was ein here: \\ sine dinc stunden mit erin \\ unde mit grozen zuhtin an sinen hove \\ $[\ldots]$ \\ daz ime da an gote nichtes ne gebrach, \\ wene daz er ane vrowen was (KR 13-18). ${ }^{54}$
}

Die kreisförmige Handlungsführung wird über die Tugend der Ehre zusammengeschnürt, die nun wiederhergestellt ist. Dabei belässt es der Text aber nicht, sondern steigert die Vorbildlichkeit zusätzlich dadurch, dass er sie am Ende als

51 | Vgl. dazu auch Borgolte/Jaspert (2016: 23), die notieren: "Auf das Meer hinaus zu fahren stellte für den Menschen stets ein Wagnis dar. Die See darf als die archetypische Gefährdung des Menschen gelten".

$\mathbf{5 2} \mid$ "[I]n ihr Heimatland".

53 | S. dazu den bereits zitierten Vers dîn wif ist wider over mere (KR 3302), der das Meer deutlich als Barriere zur 'kulturellen Anderwelt‘, sprich hier zur Welt Konstantins, ausweist.

$\mathbf{5 4} \mid$ "Rother war ein (wahrer) Fürst: Seine Hofhaltung war angesehen und entsprach den gesellschaftlichen Vorstellungen [...] so daß es inm an nichts Erstrebenswertem fehlte, außer, dass er keine Königin hatte." 
relationale Überlegenheit präsentiert, die (wie bereits im Vergleich der Erzählwelten dargestellt) auf den kommunikativen Handlungsmaximen des Königs beruht. Diese werden nun noch einmal in breiter Öffentlichkeit zur Schau gestellt, wenn Rother durch intensive Beratung über den richtigen und richtenden Umgang mit dem unterlegenen Brautvater befindet (vgl. KR 4613-4646).

Die zunächst vergleichende Betrachtung verschiedener Kulturmuster wird in diesem Ausgang damit in eine hierarchische Stufung, die Kontingenz divergierender, möglicher Positionen in historische Notwendigkeit überführt und damit auch (kulturelle) Vorbildlichkeit klar definiert. Dabei wird das Ende bereits im Verlauf der Handlung implizit vorweggenommen: So verweist die ebenfalls namenlose Gattin Konstantins beständig auf die Schwächen des Herrschers, die insbesondere an dessen Maßlosigkeit (overmut, $K R$ 4562) festgemacht werden. Das Konzept der Maßlosigkeit, das einem heldenepischen Ethos entspringt, ${ }^{55}$ erfährt hier eine deutliche Abwertung und markiert so die Grenze zu einer rohen, unzivilisierten Welt der Gewalt: dinis overtruwen scaden, / ich ne mochtis dir zende nie gesagin (KR 4549), ${ }^{56}$ schilt die Königin Konstantin. Es tangiert und charakterisiert auch das Kommunikationsverhalten Konstantins gegenüber seiner Gattin, das sich jeder konsiliarischen Einflussnahme verschließt: owi, we gerne ich noch riete (KR 1182), ${ }^{57}$ bekennt die Königin und empfiehlt sich damit zwar als Beraterin, unterstreicht durch die Wahl des Konjunktivs zugleich aber auch die Zwecklosigkeit eines solchen beratenden Bemühens. Die Königinmutter ist eine hybride Figur, indem sie in einem Reich beheimatet ist, dessen Werte sie nicht vertritt, oder anders herum, indem sie bereits an die westliche Zielwelt Rothers angenähert wird, der sie zunächst noch nicht angehört. Dies zeigt sich auch, wenn sie durch ironische Äußerungen Distanz zu den Handlungen Konstantins einnimmt: Die bereits angeführte Szene mit dem Löwen, der seinen Tod an der Saalwand findet, kommentiert sie in für den Gatten äußerst demütigender Manier:

"nu warte", sprach sie, "wie genir hoveman

din vederspil irzogen hat,

der da vor deme dische stat!" (KR 1175-1177) ${ }^{58}$

Aber auch Rothers Gefolgsleute betrachten sie bald schon als Verbündete, wenn etwa Berchter aus ihrer Gesinnung (freudige) Zuversicht schöpft: ich troste mich an de kunigin! $(K R 1224)^{59}$

55 | VgI. zur Abgrenzung der Verhaltensform des 'Barbarischen` gegenüber dem ^Höfischen ، auch: Ehrismann 2005: 80f.; sowie zum Barbarischen: Scheibelreiter 1999.

56 | "Den Schaden, den deine überhebliche Sturheit angerichtet hat, vermochte ich dir ja niemals zur Gänze klarzumachen«.

57 | "Ach, wie gerne wollte ich dir jetzt noch raten".

$\mathbf{5 8} \mid$ 'Schau dochı, sprach sie, 'wie jener Höfling dein Jagdvögelchen gezähmt hat, (jener Höfling,) der dort vor der Tafel steht! «

59 | "Die Königin macht mich zuversichtlich!“ 
Mit der (impliziten) Vorausdeutung wählt der Rother ein Erzählschema, das der Heldenepik vertraut ist, das er aber - gerade entgegen der heldenepischen Umsetzung - nicht konsequent bis zum Schluss verfolgt. ${ }^{60}$ Denn das Rother-Ende zeugt von dem Versuch eines bewussten Bestehen-Lassens beider (also der in der Kontaktrelation spezifizierten) Kulturen. Mit der translatio per nuptias (vgl. Gellinek 1968: 82) geht der kaiserliche Herrschaftsanspruch Ostroms zwar auf Rother über und manifestiert damit eine hierarchische Form des In-BeziehungSetzens beider Reiche, zugleich wird jedoch verhindert, dass dem Anderen gänzlich die Existenz entzogen würde. Dies bekundet sich auch im abschließenden Urteil Rothers über Konstantin, in dem der Herrscher - obwohl er die richtende Gewalt innehat und entscheiden kann, wie mit dem Unterlegenen verfahren wird - sein vorhandenes Machtpotenzial nicht ausspielt. Er bezeugt vielmehr zuchte unde erin ( $\left.K R{ }_{4}{ }_{617}\right)$, also ein fürstliches Verhalten, wenn er Konstantin eine gewaltfreie Behandlung gewährt und auch über dessen Reich Byzanz keinen Herrschaftsanspruch erhebt. ${ }^{61}$ So ist zu fragen: Weshalb endet der kulturelle Kontakt im Rother mit einem derart versöhnlichen Ausgang und eben nicht, dem Erzählmuster der Chanson de Geste entsprechend, mit der kämpferischen Vernichtung des Gegenübers? Warum wird die fremde Figur nicht stärker beschädigt, warum ihre Welt nicht mit der Hochzeit geschlossen?

Das gewählte Ende, so ließe sich mit Monika Schulz eine erste Antwort finden, begründet die singuläre Liebesbeziehung auf der Handlungsebene, indem sie die Ehe nach allen Seiten hin legitimiert, insbesondere, da sie nach östlichem Recht auch der Zustimmung des Brautvaters, nicht nur des Ehemanns, bedarf (vgl. Schulz 2005: insb. 27-54). Doch allein über diese eherechtliche Konvention $\mathrm{zu}$ argumentieren vermag angesichts der breit angelegten und mit großem erzählerischen Aufwand gestalteten Schlussinszenierung nicht zu genügen. Vielmehr scheint damit ein über die Handlungsebene hinausgehendes Plädoyer verbunden. Ungeachtet der ausgestellten kulturellen Überlegenheit eines (des Rother'schen) Herrschaftsmodells votiert der KR für ein Zulassen bzw. Aushalten von Differenz. Dass diese Differenz beidseitig anerkannt wird, kulminiert in der finalen Gemütsregung des königlichen Kontrahenten Rothers, der diesem schlussendlich mit Akzeptanz begegnet: Rotheres ere was ime lief (KR 4756f.). ${ }^{62}$ Die wiederhergestellte Ehre Rothers wird damit nicht nur zusätzlich von außen bezeugt, sondern zum symbolischen und geteilten Band der Ehre, das den Gegner umschließt. Im erzählerischen Gesamtkontext vermag es auch als

60 Auch Schmitz notiert, dass die epischen Vorausdeutungen "die Dichtung nur zum Teil mit der Heldenepik assoziierbar machen" (Schmitz 2002: 175, Anm. 4). Reiffenstein verweist diesbezüglich insbesondere auf die positiven Vorausdeutungen im Rother, die zukünftige Freude ankündigen und sich von den heroischen Vorausdeutungen im Nibelungenlied unterscheiden (vgl. Reiffenstein 1972: 556f.).

61 | Vgl. dazu auch Kohnen (2014: 229), die betont, dass Konstantins Herrschaft unverletzt bleibt und sogar der Status seiner Frau als mächtige Königin am Ende bekräftigt würde.

62 | "Rothers Ansehen war auch sein Anliegen geworden ". 
(Werte-)Basis zu fungieren, die die Voraussetzung eines möglichen Bündnisses der beiden christlichen Reiche (gegen den Islam bzw. die Heiden) schaffen könnte. ${ }^{6}$ Im Rahmen christlicher Einheit wird hier das Anerkennen gewisser kultureller und herrschaftlicher Vielfalt als ein höheres vergemeinschaftendes Prinzip geltend gemacht.

\section{Funktionalisierung der Brautwerbung ALS INTERKULTURELLES KONTAKTSCHEMA}

Aus diesen Folgerungen leiten sich wesentliche Erkenntnisse zur Beantwortung des dritten Fragekomplexes ab, der sich damit befasst, welches Potenzial und Verständnis dem Text zukommt, wenn die Interkulturalität als festes Strukturelement gezielt in die Analyse einbezogen wird.

Das Werkende bestätigt nicht allein das narrative Erzählmuster der Brautwerbung, sondern begründet den moralischen Vorrang eines (Herrschafts-)Modells, das sich erst über den interkulturellen Kontakt als solches hervortut. Denn kulturelle Überlegenheit wird hier nicht dadurch gefestigt, dass das Eigene programmatisch aufgerufen und so stabilisiert würde, sondern tritt erst in der Konfrontation mit dem Anderen als vorbildlich hervor. ${ }^{64}$ Der (exkludierende) Dualismus von vertrauter Welt und bedrohlicher Fremde wird folglich nicht, wie für andere Wissenssysteme des Mittelalters kennzeichnend, ${ }^{6} 5$ bloß wiederholt, sondern dialogisiert. Dieser Perspektive ist ein spezifisches Verhältnis von eigen und fremd zu entnehmen: Es bindet die Fremde in eine argumentativ-logische Struktur zur Behauptung des Eigenen ein, macht sie zur Voraussetzung, um das Eigene besser sichtbar zu machen.

Mit dem Brautwerbungsschema gelingt es, interkulturellen Kontakt abzubilden, der (aus der Notwendigkeit der Sicherung des genealogischen Fortbestandes) das Andere in den Blick nimmt, es als Ort der Bewährung voraussetzt, hinterfragt und problematisiert (vgl. dazu auch Röcke 1991: 41). Indem diesem Anderen etwas (nämlich die Braut) >entnommen` wird, kann es weder, wie im Erzählmuster der Heldenepik üblich, als völlig >Außerhalb<, als zu >Vernichten-

63 | Der interreligiöse Konflikt, der die mittelalterliche Welt fundamental bewegte, schwelt im Rother aber eben nur im Hintergrund (s. auch Anm. 69).

64 | Ähnlich auch Röcke: "Eine grundsätzliche Neubestimmung des antithetischen Verhältnisses von Eigenem und Fremdem hingegen ist erst dann festzustellen, wenn die Fremde [...] zum Darstellungsmodus, ja zur Voraussetzung des Eigenen stilisiert wird, z.B. zur Voraussetzung individueller Bewährung oder individueller Wünsche" (Röcke 1991: 43).

65 | Diese Veränderung in der Wahrnehmung des Fremden zeigt sich auch im Vergleich mit Pilgerberichten nach Jerusalem, wie sie seit dem Hochmittelalter in großer Zahl vorliegen: Diese blenden "die durchreiste Fremde zu einem erheblichen Teil aus" und richten den Fokus allein auf die Heilsgeschichte und -bedeutsamkeit der heiligen Stätten für die Pilger (Münkler 2002: 329f.). 
des-Anderes < betrachtet noch durch Streichung aus der Geschichte und damit der Argumentation ausgegliedert werden. Das Brautwerbungsschema bildet ein narratives Muster, um moralische, kulturelle und herrschaftliche Überlegenheit anders, argumentativ-diplomatisch, durch die Entfaltung der Idealität eines (des weströmischen) Reiches, nicht aber in primär konfrontativer Weise, im Bestreben nach kämpferischer, kriegerischer Machtausübung zu demonstrieren. ${ }^{66} \mathrm{Zu}$ gleich präsentiert es die Höfe als kommunizierende Organe im Ganzen der mittelalterlichen >europäischen $<$ Gesellschaft.

Anstatt zu argumentieren, die Schemata und Motive im $K R$ würden »durch >sinnlos< machende Übersteigerung des Gebrauchs ad absurdum geführt« (Stein 1988: 334f.), kann die auffällige Vervielfältigung tradierter Muster vielmehr als bewusste Gestaltungsmaßnahme ${ }^{67}$ verstanden werden, mit der sich eben doch ein erkennbares Sinnpotenzial verbindet: Der so inszenierte, repetitive Erzählfluss verdeutlicht, dass sich das Vorbildliche, Erstrebenswerte nur in einem fortwährenden, wiederholten Kontaktprozess zu etablieren, zu konturieren und als vorbildlich hervorzutun vermag. Diesen Kontaktprozess unter dem Schlagwort der Interkulturalität näher zu beleuchten, kann auch unter Verweis auf Weinberg (vgl. 2014) nur befürwortet werden, der Kritik an der häufig mit den Begrifflichkeiten einhergehenden (epochalen) Skopuseinschränkung übt: Weinberg betont dabei zu Recht, dass das Konzept der Transkulturalität nicht an einer zeitlichen Entwicklung orientiert ist, weil »Kulturen [...] schon damals nicht nach einem [...] Kugelmodell funktioniert haben«, ihre Kulturkonzepte damals bereits »deutlich komplexer« waren (ebd.: 9). Solche größere Komplexität lässt sich folglich nicht als Charakteristikum der globalisierten Moderne deuten, sondern war »in genuin interkulturellen Räumen immer schon zu konstatieren« (ebd.: 19). Das Weinberg'sche >damals<, das den Blick insbesondere auf das 18./19. Jh. lenkt, bestätigt der $K R$ in diachroner Verlängerung für das Mittelalter ebenfalls. Texte wie der $K R$ erfassen in narrativer Umsetzung kulturelle Komplexität, indem sie den Kontakt zwischen Welten initiieren, fremde Herrschaftsräume öffnen und damit zur Darstellung bringen.

Mittelalterliche Texte aus interkultureller Perspektive zu lesen erfordert allerdings, wie am KR deutlich wurde, ein weites Kulturverständnis, das Interkulturalität nicht allein auf die Thematik >Christen versus Heiden < beschränkt. Auf dieser Basis konnte gezeigt werden, wie anhand des konventionellen Erzählmodells Brautwerbung bzw. dessen Variation interkulturelle Kontakte erzählerisch dargestellt wurden. Es gewährt Einsicht in die Differenz von Herrschafts- und Gesellschaftsformen auf einer Ebene jenseits einer rein theoretisch-abstrakten Behandlung des Phänomens.

66 | Mit Bezug auf die Deutung des Geschehens im zeitgenössischen Kontext ließe sich die Ausstellung der moralischen, intellektuellen Überlegenheit auch als Reaktion auf die Erfahrungen der Kreuzzüge beziehen, die den Westen mit einer vor allem materiell überlegenen Kultur des Orients konfrontierte (vgl. dazu Brunner 2017: 139).

67 | Dazu auch Kiening (1998: 221): "Die Distanz zum Schema hat einen neuen Grad" erreicht. 
Mit der im Rother-Ende zudem bezeugten Akzeptanz des Anderen tritt der Text ins Spektrum jener mittelhochdeutschen Epen, die von interkultureller Toleranz erzählen. Er lässt sich zum einen als Variante zum (eingangs genannten) Wolfram'schen Willehalm begreifen. Als Gegenmodell zum Rolandslied verweist er zum anderen darauf, dass mittelhochdeutsche Texte eben nicht nur den Modus >Kultur/Un-Kultur < (wie er hier klassisch vertreten wird) kennen, sondern auch über das >Kultur/Kultur-Schema< verfügen. Dabei ist der Rother (im Rahmen der Interkulturalität) so bedeutsam, weil er jenseits der üblichen Abgrenzungsparadigmen operiert, die primär auf religiös (Christen versus Heiden), sozial (höfisch/städtisch versus bäuerlich) oder sprachlich (welsch versus tiutsch) binären Mustern basieren. Auch der Rother erzählt von einem Religionskonflikt, der in der Behauptung des Christentums gegenüber dem Islam kämpferisch ausgetragen wird, und zeigt damit, dass ihm die konventionellen Distinktionen durchaus vertraut sind. Indem er sie jedoch in den Nebenstrang auslagert, ${ }^{68}$ tritt umso deutlicher hervor, dass er den Fokus auf die Verhandlung kultureller Differenzen, Konflikte und Vergleiche innerhalb einer christlichen Welt legt. Die Forschung kann er damit dazu anregen, das bisher vorrangig anvisierte Spektrum der als interkulturell beschriebenen Werke auszubauen und in andere Richtungen weiterzudenken. Der $K R$ bildet in dieser Lesart nicht nur die den mittelalterlichen Text beherrschende Diskussion um divergierende Kommunikationsmodelle in verschiedenen kulturellen Kontexten ab, sondern weist damit gleichfalls in aktuelle gesellschaftspolitische Zusammenhänge. ${ }^{69}$

68 Der Konflikt mit Ymelot tangiert die Brautwerbung nur peripher und bildet nicht das Zentrum der interkulturellen Spannungen zwischen den Protagonisten Rother und Konstantin. Beide Herrscher sind an einer Bekämpfung des Heidenführers Ymelot interessiert.

69 | Diese Aktualität zeigt sich etwa in der beeindruckenden Zahl von zeitgenössischen Theaterinszenierungen, die Kommunikation (bzw. Nichtkommunikation, Kommunikations- oder Sprachstörung) zum Thema haben und mit denen sich auch das Verlangen zu artikulieren scheint, Formen politisch-gesellschaftlicher Sprache, des Miteinandersprechens wieder stärker in den Fokus zu rücken. Beispielhaft wäre auf die Vielzahl an Adaptionen antiker Stoffe, wie der Medea, zu verweisen, die zeigen, was passiert, wenn der Kompromiss (von dem eine demokratische Gesellschaft lebt) verweigert wird und Sprachlosigkeit bzw. Sprachverweigerung und Verweigerung von Sprachkontakt in die Tragödie führt. Warum in diesem Zusammenhang (und mit etwas mehr Optimismus als im antiken Mythos) also nicht auch einmal den mittelhochdeutschen Rother auf die Bühne bringen? 


\section{LITERATUR}

Bauer, Matthias (2005): Romantheorie und Erzählforschung. Eine Einführung. Stuttgart.

Borgolte, Michael/Jaspert, Nikolas (2016): Maritimes Mittelalter - Zur Einführung. In: Dies. (Hg.): Maritimes Mittelalter: Meere als Kommunikationsräume. Ostfildern, S. 9-34.

Bumke, Joachim (1979): Mäzene im Mittelalter. Die Gönner und Auftraggeber der höfischen Kultur in Deutschland. 1150-1300. München.

Brunner, Horst (2017): König Rother. In: Ders. (Hg.): Geschichte der deutschen Literatur des Mittelalters und der Frühen Neuzeit im Überblick. Stuttgart, S. 138-140.

Cassirer, Ernst (1990): Versuch über den Menschen. Einführung in eine Philosophie der Kultur. Hamburg.

Ders. (2010): Philosophie der symbolischen Formen. Hamburg.

Colin, Nicole (2013): Im toten Winkel der Versöhnung. Mittler wider Willen im deutschfranzösischen Kulturtransfer. Der Fall Jean Villar. In: Zeitschrift für interkulturelle Germanistik 4, H. 2, S. 95-110.

Czerwinski, Peter (1989): Exempel einer Geschichte der Wahrnehmung. Bd. 1: Der Glanz der Abstraktion. Frühe Formen von Reflexivität im Mittelalter. Frankfurt a.M.

Ebenbauer, Alfred (1984): Es gibt ain mörynne vil dick susse mynne. Belakanes Landsleute in der deutschen Literatur des Mittelalters. In: Zeitschrift für deutsches Altertum und Literatur 113/1, S. 16-42.

Ehrismann, Otfrid (2005): Das Nibelungenlied. München.

Fromm, Hans (1976): Die Erzählkunst des Rother-Dichters. In: Walter Johannes Schröder (Hg.): Spielmannsepik. Darmstadt, S. 351-376.

Geertz, Clifford (1987): Dichte Beschreibung. Beiträge zum Verstehen kultureller Systeme. Übers. v. Brigitte Luchesi u. Rolf Bindermann. Frankfurt a.M.

Gellinek, Christian (1968): König Rother. Studie zur literarischen Deutung. Bern.

Haupt, Barbara (2009): Heidenkrieg und Glaube. Zur Toleranz im Willehalm Wolframs von Eschenbach. In: Bernd Springer (Hg.): Religiöse Toleranz im Spiegel der Literatur. Zürich, S. 41-56.

Jackson, Timothy R. (1969): Religion and love in Flore und Blanscheflur. In: Oxford German Studies 4, S. 12-25.

Jolie, Stephan (2005): Gewalt - Text - Ritual. Performativität und Literarizität im König Rother. In: Beiträge zur Geschichte der deutschen Sprache und Literatur 127, S. 183-207.

Kiening, Christian (1998): Arbeit am Muster. Literarisierungsstrategien im König Rother. In: Wolfram-Studien 15, S. 211-243.

König Rother (2000): Mittelhochdeutscher Text und neuhochdeutsche Übersetzung von Peter K. Stein. Hg. v. Ingrid Bennewitz. Stuttgart (zit. als KR).

Kohnen, Rabea (2014): Die Braut des Königs. Zur interreligiösen Dynamik der mittelhochdeutschen Brautwerbungserzählungen. Berlin.

Martínez, Matías/Scheffel, Michael (2012): Einführung in die Erzähltheorie. 9., akt. u. überarb. Aufl. München. 
Meves, Uwe (1976): Studien zu König Rother, Herzog Ernst und Grauer Rock (Orendel). Frankfurt a.M.

Münkler, Marina (2002): Alterität und Interkulturalität (ältere deutsche Literatur). In: Claudia Bentien/Hans Rudolf Velten (Hg.): Germanistik als Kulturwissenschaft. Eine Einführung in neue Theoriekonzepte. Reinbek b. Hambrug, S. 323-344.

Ort, Claus-Michael (2003): Kulturbegriffe und Kulturtheorien. In: Ansgar Nünning/Vera Nünning (Hg.): Konzepte der Kulturwissenschaften. Stuttgart, S. 19-38.

Reiffenstein, Ingo (1972): Die Erzählervorausdeutung in der frühmittelhochdeutschen Dichtung. Zur Geschichte und Funktion einer poetischen Formel. In: Herbert Backes (Hg.): Festschrift für Hans Eggers zum 65. Geburtstag. Tübingen, S. 551-576.

Röcke, Werner (1992): Schreckensort und Wunschwelt. Bilder von fremden Welten in der Erzählliteratur des Mittelalters. In: Der Deutschunterricht 44/2, S. 32-48.

Samples, Susann (2001): Belacane. Other as Another in Wolfram von Eschenbach's Parzival. In: Fiona Wheeler/Bonni Tolhurst (Hg.): On Arthurian Woman. Essays in memory of Maureen Fries. Dallas, S. 187-198.

Scheibelreiter, Georg (1999): Die barbarische Gesellschaft. Mentalitätsgeschichte der europäischen Achsenzeit 5.-8. Jh. Darmstadt.

Schmid-Cadalbert, Christian (1985): Der ,Ortnit AW، als Brautwerbungsdichtung. Ein Beitrag zum Verständnis mittelalterlicher Schemaliteratur. Bern.

Schmitz, Silvia (2002): Erzählen im 'König Rotherı. In: Ludger Lieb/Stephan Müller (Hg.): Situationen des Erzählens. Aspekte narrativer Praxis im Mittelalter. Berlin, S. 167-190.

Schott, Clausdieter (1986): [Art.] "Ehe. B. Recht. VI. Germanisches und deutsches Recht». In: Lexikon des Mittelalters TI. 3 p., Sp. 1629-1630.

Schotte, Manuela (2009): Christen, Heiden und der Gral. Die Heidendarstellung als Instrument der Rezeptionslenkung in den mittelhochdeutschen Gralromanen des 13. Jahrhunderts. Frankfurt a.M.

Schulz, Monika (2005): Eherechtsdiskurse. Studien zu König Rother, Partonopier und Meliur, Arabel, Der guote Gêrhart, Der Ring. Heidelberg.

Stein, Peter (1988): Do newistich weiz hette getan. Ich wolde sie alle ir slagen hanc. Beobachtungen und Überlegungen zum König Rother. In: Ders./Renate Hausner/ Ingo Reiffenstein (Hg.): Festschrift für Ingo Reiffenstein zum 60. Geburtstag. Göppingen, S. 309-338.

Szklenar, Hans (21985): [Art.] "König Rother». In: Burghart Wachinger u.a. (Hg.): Die deutsche Literatur des Mittelalters. Verfasserlexikon. Bd. 5: Kochberger, JohannesMarien-ABC, Berlin/New York, Sp. 82-94.

Weinberg, Manfred (2014): Die Grenzen der Interkulturalität. In: Ingeborg Fiala-Fürst (Hg.): Prager deutsche, deutschböhmische und deutschmährische Literatur. Eine Neubestimmung. OImütz, S. 7-32. 